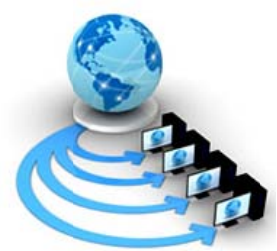

Volume 9, No. 1, January-February 2018

International Journal of Advanced Research in Computer Science

RESEARCH PAPER

\title{
UNDERWATER IMAGE ENHANCEMENT USING DWT BASED MEAN IMAGE FUSION RULE
}

\author{
Sharanjit Kaur \\ Research Scholar: Computer Science \& Engineering, \\ SUS College of Engineering \& Technology, Tangori, \\ Punjab (India)
}

\author{
Er. Deepinder Kaur \\ Assistant Professor: Computer Science \& Engineering, \\ SUS College of Engineering \& Technology, Tangori \\ Punjab, India
}

\begin{abstract}
Due to the physical properties of water and its environment, underwater image processing has received a considerable attention since last decade. Underwater activities in discovering and recognizing objects have resulted in new challenges. Consequence from these activities, significant problems have raised due to the light absorption and diffusion effects. As light travels in water, a rapid exponential loss of light intensity occurs depending on the color spectrum wavelength. In this work, Transform based method has been used to preserve the contrast and color of the images. At first, Color and contrast corrected images has been evaluated using HSV color space and CLAHE method of adaptive histogram equalization. Then these two images has been fused together using different fusion rules at two level decomposition using DWT. Different fusion rules has been explored in which mean od approximation and detailed coefficient found effective than the rest. It gives high PSNR values along with improved contrast and entropy of the image. Experimental results have been compared between different methods of fusion by PSNR, MSE, Average gradient, entropy and contrast parameters. Proposed method has been tested on number of images and results found at various stages of the algorithm have been displayed. In future, similar work can be explored further by taking different transform based decompositions as only DWT has been tested in this work. Also Different wavelet families have been explored during work and 'Bior6.8' found to be effective one than the other.
\end{abstract}

Keywords: CLAHE, wavelettransform,underwater image enhancement, image fusion etc.

\section{INTRODUCTION}

The invention of computers is the pillar for modern science and technology. Digital image processing is a predominant area, which is based on computer technology. Digital image processing supports a wide range of applications ranging from daily needs like mobiles, laptops, photography to high level research fields like medicine, satellites, radars, remote sensing, etc. With the development of technology and invention of several image-capturing devices, millions of new images are being captured daily, which need automatic processing and manipulation [1].

All image acquisition devices like satellites, radars, cameras have one thing in common, i.e., sensors. As shown in figure 1.1, image acquisition by the digital sensors is the initial step in image processing. In some scenarios, data acquired by the sensors at high speed results in abnormal content, which needs processing to perceive the visual content by the human eye in a pleasant way. In 1960s the processing of the digital image was considered to be an expensive affair because of the expensive hardware used in the process. Digital image sensors have witnessed revolutionary changes in the past few decades and the hardware cost has drastically reduced, which made digital image processing applications to be used widely in different research fields. Digital image processing has various advantages over the analog image processing, which produces highest quality digital images.

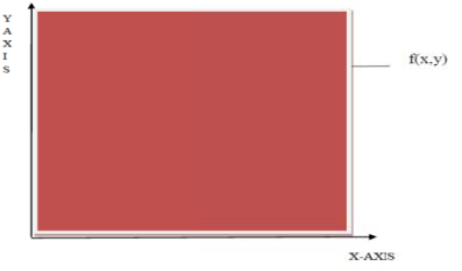

Figure 1: Digital image [2].

As shown in figure 1.2, a digital image is represented technically as a two dimensional function $\mathrm{f}(\mathrm{x}, \mathrm{y})$, which represents the intensity of selected pixel. Here $f$ denotes the intensity and (x,y) is termed as the weight of the pixel, which gives the exact location of pixel in the digital image.

\section{LITERATURE SURVEY}

YANG et al. [3] provide an enhancement method for underwater laser imaging system by blind de-convolution method based on small angle approximation. Also introduced a modified normalized mean square error (NMSE) method to validate the convergence of the blind deconvolution algorithm, Use modulation transfer function (MTF), point spread function (PSF).

Shahrizan et al .[4] contrast correction technique is applied to the image usimg modified Von Kries hypothesis and stretching the image into two different intensity images at the average value with respects to Rayleigh distribution. Also color correction technique is applied to the image where the image is first converted into hue-saturation-value (HSV) color model and then RGB color.

Tingting et al.[5]Improved images in terms of brightness and color fidelity. Used image structural decomposition for image enhancement, histogram equalization and 
Retinexalgorithms are applied for non-uniform brightness correction of images.

Banerjee et al. [6]Did Underwater image enhancement; anisotropic diffusion; color cast; CLAHE; linear and nonlinear filters. Compared with three golden methods, namely, Gray World (GW), White Patch (WP), Adobe Photoshop Equalization (APE)

\section{PROPOSED WORK}

The proposed enhancing strategy consists of three main steps: color correction (first input of fusion process) in, contrast enhancement (second input of fusion process) and multi-scale fusion process for the two inputs. In multi-scale fusion process, wavelet decomposition and reconstruction as well as fusion principles for low frequency and high components will be introduced.

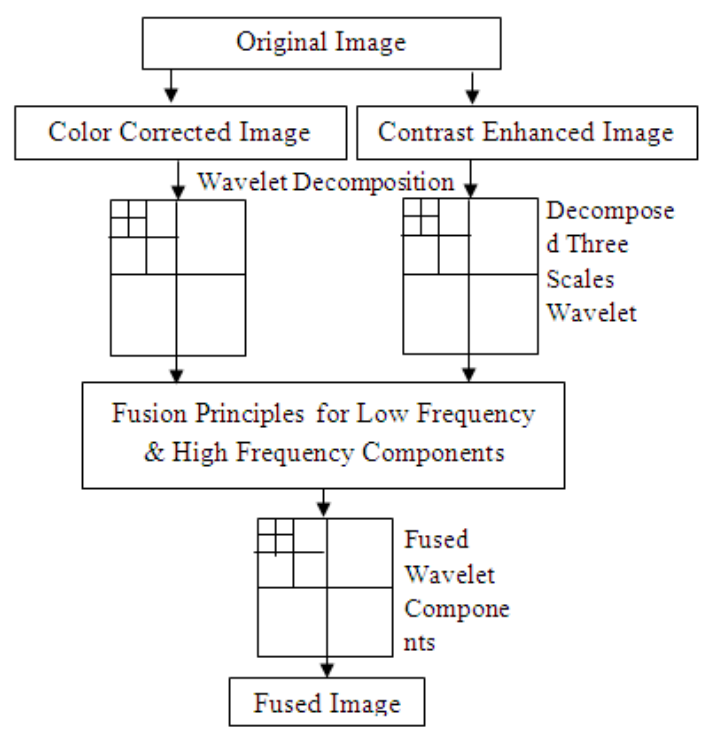

Figure 2: The steps for underwater image enhancement based on wavelet transformation

As local variance method has been adopted for fusion in the existed work, other methods has been explored based on minimum, maximum and mean values of detailed and approximation coefficients. In this bi-orthogonal wavelet is also explored which gives better PSNR value than Haar wavelet. The main algorithm which has been used in the proposed work has been detailed as below.

\section{- Color Correction}

Underwater imaging is influenced by non-uniform color cast due to absorption of the propagated light. Color cast corresponds to the varying degrees of light attenuation. Different wavelengths of light are attenuated at different rates in water. In our experiments, a simple and efficient white balance operation is used to enhance the image appearance by discarding color casts. The proposed white balance algorithm based on the Shades of Grey [7] with gain factor is more computationally effective.

- Contrast Enhancement

Despite the color casts caused by light absorption, underwater images are also affected by low contrast due to backward scattering of light. Contrast enhancement is widely utilized in image processing because it brings out more details of images.

\section{- Multi-scale Fusion Process}

In the existed fusion framework, the first input is color corrected image and the second input is contrast enhanced image. Each input is decomposed into low frequency and high frequency components by applying the wavelet operator to three scales. Then, different fusion principles are utilized to fuse the low frequency and high frequency components. Weighted average is enforced to fuse low frequency components, while local variance is employed in the fusion of high frequency components. After the fusion process, the new low frequency and high frequency components are generated. Finally, the enhanced image is obtained by reconstructing the new low frequency and high frequency components.

\section{RESULTS AND DISCUSSIONS}

In this work, different fusion rules has been proposed based om average and maximum property of the coefficients of DWT.Theresults in terms of output images at different steps have been described below.

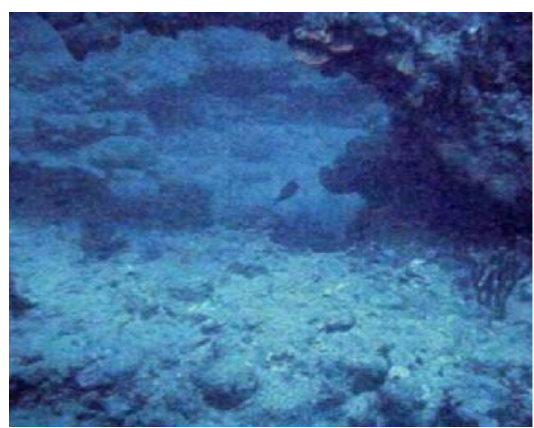

Figure 3: Input underwater image

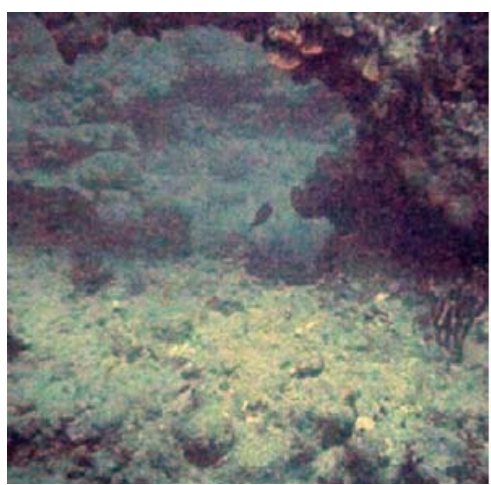

Figure 4: Color corrected image

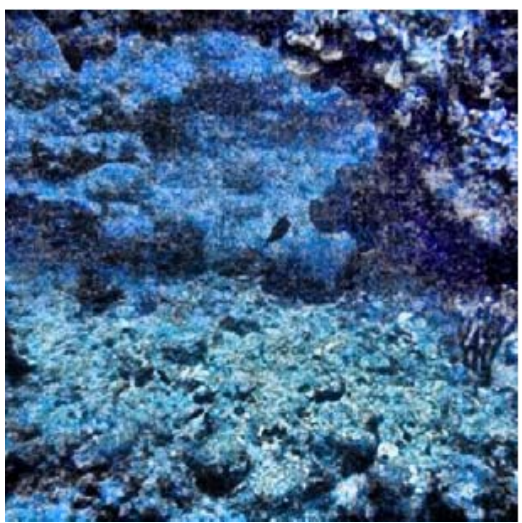

Figure 5: Contrast corrected image 


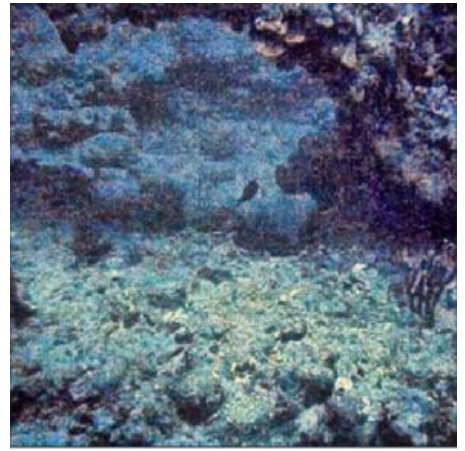

Figure 6: Enhanced image using two-level DWT (bior6.8) and VSD based Fusion

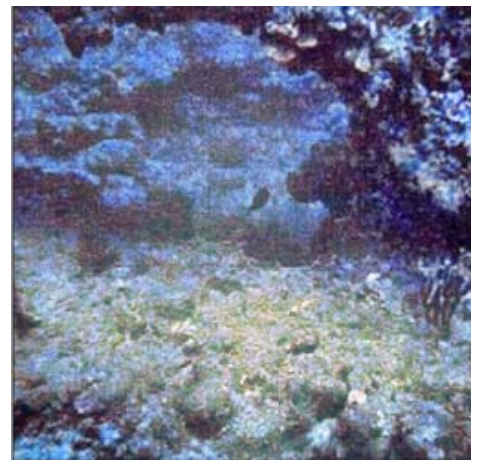

Figure 7: Enhanced image using two-level DWT (bior6.8) and Mean of DC and Max of AC

Table 1: The results by different parameters for different methods

\begin{tabular}{|l|l|l|l|l|l|}
\hline Parameters & MSE & PSNR & Gradient & Contrast & Entropy \\
\cline { 1 - 5 } Methods & 889.3069 & 18.6402 & 8.809 & 36.99 & 7.54 \\
\hline Haar and VSD based fusion & 869.4087 & 18.7385 & 8.543 & 36.92 & 7.54 \\
\hline $\begin{array}{l}\text { Bior6.8 and VSD based fusion } \\
\text { AC }\end{array}$ & 866.8885 & 18.7511 & 8.474 & 36.95 & 7.54 \\
\hline $\begin{array}{l}\text { Bior6.8 and Mean of DC and Mean of } \\
\text { AC }\end{array}$ & 847.27 & 18.8505 & 8.088 & 36.79 & 7.53 \\
\hline $\begin{array}{l}\text { Bior6.8 and Max of DC and Max of AC } \\
\text { Bior6.8 and Mean of DC and Max of } \\
\text { AC }\end{array}$ & 1524.87 & 16.2984 & 8.676 & 38.56 & 7.66 \\
\hline
\end{tabular}

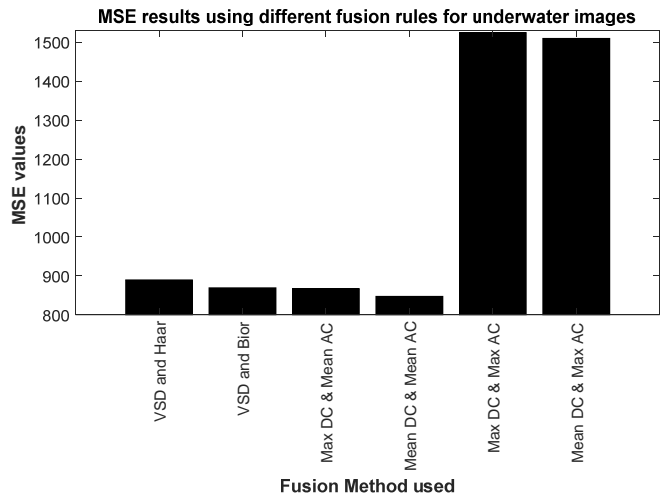

Figure 8: MSE results using different fusion rules for underwater images

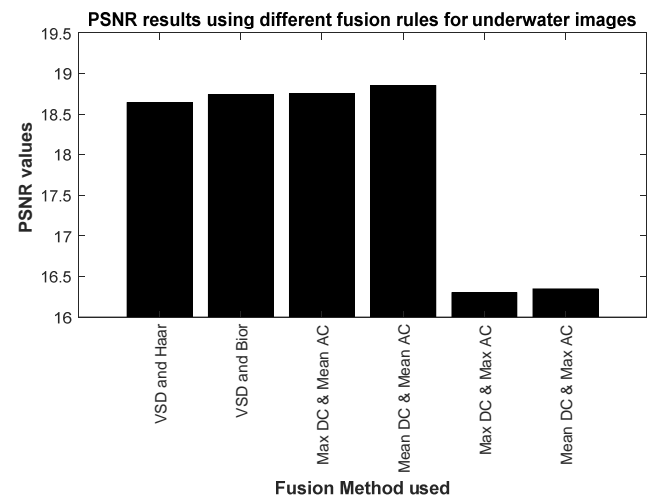

Figure 9: PSNR results using different fusion rules for underwater images

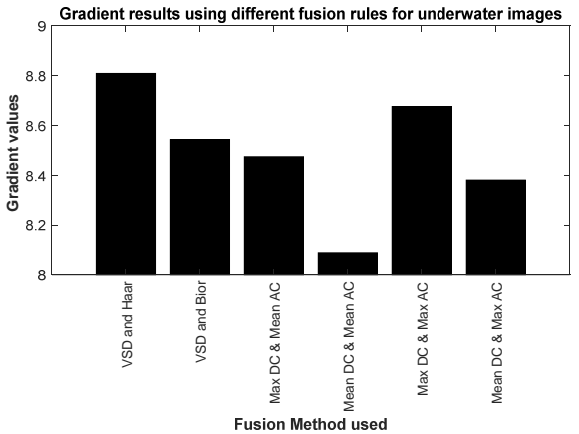

Figure 10: Average Gradient results using different fusion rules for underwater images

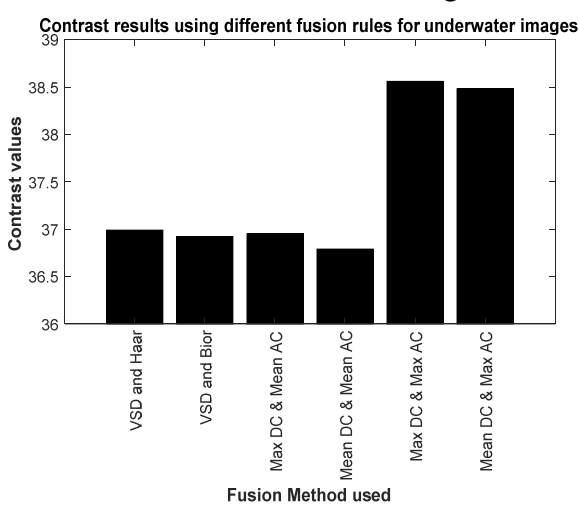

Figure 11: Contrast results using different fusion rules for underwater images 


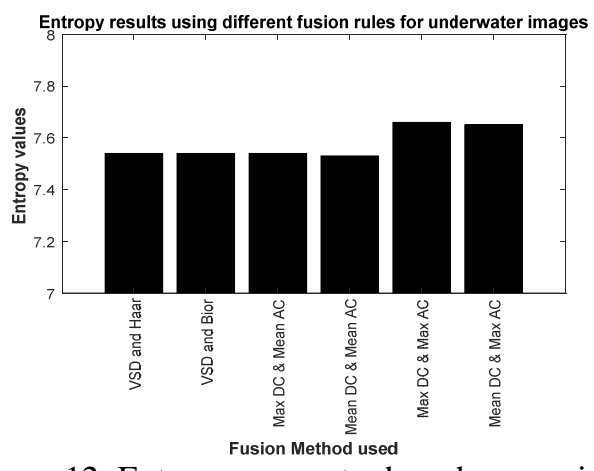

Figure 12: Entropy parameter based comparison

In the fusion process different rules have been explored using AC and DC coefficients of DWT decomposed images in which Local variance, maximum and mean value of the coefficients has been used by different combination options. Results have been tested using MSE, PSNR, Contrast, Entropy and average gradient of the enhanced images for different fusion rules. It has been found that Mean of DC and AC coefficients gives better results in PSNR value along with least average gradient as it defines the artifacts in the images after enhancement. Also it gives effective entropy and contrast values as highest entropy values preserve the content of the images whereas enhanced contrast need to be effective in such a way that more bright pixels need to be less bright and less luminance pixels need to be more values of the contrast. Below are different images which have been shown at different steps of the proposed algorithm. Generally, Original image, color corrected image, contrast corrected images and final enhanced images has been shown for a number of bad quality underwater images.

\section{CONCLUSION}

In this proposed algorithm, we used RGB image and convert it into HSV and then applied adaptive histogram equalization on luminance channel using BBHE method and the logarithm of the original image in gray scale has been applied, after this the Fast Fourier Transform has applied. After that Adds the high-pass Butterworth filter, then apply under and overexposed region enhancement using Gamma filter and saturation map and then R, G, B channel correction to achieve color correction using enhanced luminance channel and Performance is evaluated. We have also compared the result with existed popular methods i.e. histogram equalization, contrast stretching, CLAHE, BBHE, DSIHE etc. The proposed method has high PSNR value along restoring the contrast and color of the underwater input images.In this work, different fusion rules have been explored to merge color and contrast corrected images using decomposition by DWT. There are various transform based decomposition algorithms which found more effective than DWT in different research works i.e. contourlet, curve let, and shearlet etc.

\section{REFERENCES}

[1] ChiruvellaSuresh , K. AmithBansal, ”Adaptive Contrast Enhancement and White Balancing Integration for Image Enhancement Based on Non-linear Generalized Equalization Model" Published in: International Journal of Science and Research (IJSR) ISSN (Online),(2013)

[2] Rafael C. Gonzalez, Richard E. Woods, “Digital Image Processing" Published in: 3rd Edition, Prentice Hall; ISBN: 013168728X; August 2007.

[3] Fan FAN, KechengYANG , Min XIA, Wei LI, Bo FU, Wei ZHANG, " Underwater image restoration by means of blind deconvolution approach" Published in: Frontiers of Optoelectronics in China June 2010, Volume 3, Issue 2, pp 169-178

[4] Ahmad Shahrizan Abdul Ghani and Nor Ashidi Mat Isa, "Underwater image quality enhancement through composition of dual-intensity images and Rayleigh-stretching" Published in: Consumer Electronics - Berlin (ICCE-Berlin), 2014 IEEE Fourth International Conference on Date of Conference: 7-10 Sept. 2014.

[5] JI Tingting, and WANG Guoyu, "An Approach to Underwater Image Enhancement Based on Image Structural Decomposition" Published in: Journal of Ocean University of China April 2015, Volume 14, Issue 2, pp 255-260.

[6] JEET BANERJEE, RANJIT RAY, SIVA RAM KRISHNA VADALI, SANKAR NATH SHOME and SAMBHUNATH NANDY, " Real-time underwater image enhancement: An improved approach for imaging with AUV-150" Published in: Sadhana Vol. 41, No. 2, February 2016, pp. 225-238.

[7] Y. Wang, X. Ding, R. Wang, J. Zhang and X. Fu, "Fusionbased underwater image enhancement by wavelet decomposition" Published in:2017 IEEE International Conference on Industrial Technology (ICIT), Toronto, ON, 2017, pp. 1013-1018. 